\title{
QUALITY OF TRANSMISSION FOR WIFI CAMERAS NETATMO NSC01-EU, D-LINK DCS-5222L AND EDIMAX IC-7113W
}

\author{
Jan Hart, Veronika Hartova \\ Czech University of Life Sciences Prague \\ jhart@tf.czu.cz
}

\begin{abstract}
At present, we cannot do without wireless technologies. Whether they are mobile phones, WiFi networks, Bluetooth and, for example, as well as various RC models, their transmissions are ubiquitous. It takes slightly exaggeratedly, thus to define the modern era and civilization as such. One of the most commonly used bands are ISM bands. ISM bands (industrial, scientific and medical) are frequently used in many different industrial transmissions. Officially, these bands should be used exclusively for industrial, medical or scientific purposes. The FCC (Federal Communications Commission) and the ETSI (European Telecommunications Standards Institute) established the band ISM as unlicensed bands, and given that they are without royalties, so they can be widely used for commercial purposes. This article deals with the measurement of quality of wireless transmission of WiFi cameras and electromagnetic interference on the $2.4 \mathrm{GHz}$ ISM band. For this measurement the Spectrum Analyzer SPECTRAN HF-6060 and WiFi cameras D-Link DCS-5222L, Netatmo NSC01-EU and Edimax IC-7113W were used. At the defined elements wireless transmission was always measured and so that the unit is located within $3 \mathrm{~m}$ from the antenna of the spectrum analyzer SPECTRAN HF-6060; using software MSC Spectrum Analyzer wireless transmission was evaluated. Measurements defined the quality of the wireless communications and pointed to weaknesses in wireless communications of individual types of WiFi cameras. It also pointed to the risk of natural and artificial electromagnetic radiation at $2.4 \mathrm{GHz}$ ISM bands.
\end{abstract}

Keywords: WiFi, camera, interference, transmissions, wireless network, ISM band.

\section{Introduction}

At present, we cannot do without wireless technologies. Whether they are mobile phones, WiFi networks, Bluetooth and, for example, various RC models, their transmissions are ubiquitous. It takes slightly grandly, thus defining the modern era and civilization as such. Unfortunately, it is associated with less popular, but true reality and that is that if wireless transmissions are higher, it is becoming as well known electromagnetic interference. Designation of electromagnetic interference is not so accurate and so is the term used natural and artificial electromagnetic interference. This interference can affect wireless transmission and can even totally put it out of operation and therefore it is always important to determine the strength of the signal and its interference in the area.

Natural electromagnetic interference also arises from seemingly innocuous devices, such as home appliances, machinery, urban environment and high voltage lines. Artificial interference is caused deliberately. This targeted interference is used to prevent wiretapping, to prevent remote launch bombs, and also for illegal activities, such as avoiding locking the car, or jamming communications in wireless Intrusion an Hold-up Alarm Systems [1-4].

ISM bands (industrial, scientific and medical) are widely used in many different industrial transmissions. Officially, these bands should be used exclusively for industrial, medical or scientific purposes. The FCC (Federal Communications Commission) and the ETSI (European Telecommunications Standards Institute) established the band as a band unlicensed ISM and given that there are no royalties, it can be widely used for commercial purposes [3;5].

CCTV systems are one of the cornerstones of security systems. Their record is often used as a subject of lawsuits. It is therefore important that the recording always arrives from the camera to the recording device without any problems. Natural electromagnetic interference can prevent data transmission via $\mathrm{WiFi}$ or substantially endanger its resulting quality. It is therefore necessary to monitor and test individual types of wireless WiFi cameras. It is essential that the transmission reliability of WiFi cameras be detected in time.

Therefore, the goal was to measure wireless transmissions with today's most widely used WiFi cameras. These cameras have been selected on the basis of consultation with the premier distributors in the Czech Republic. 


\section{Materials and methods}

A SPECTRAN HF-6060 spectrum analyzer (Fig. 1) was used for measurements to investigate the quality of wireless transmissions regarding WiFi cameras and electromagnetic interference at a frequency of $2400 \mathrm{MHz}$, which is among the ISM bands.

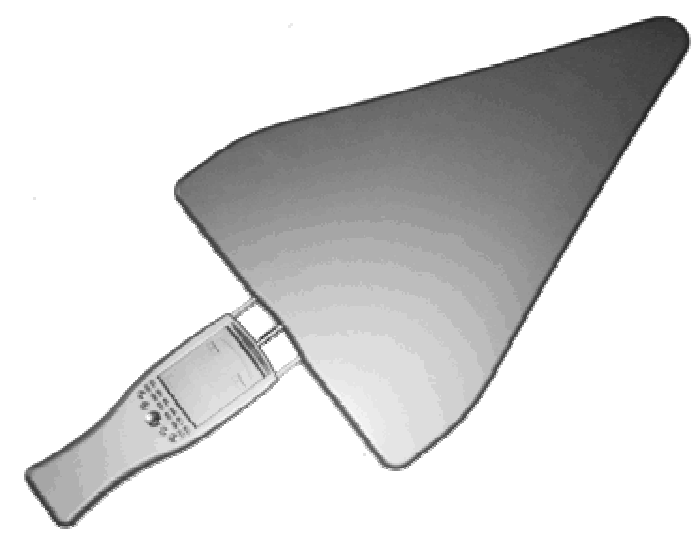

Fig. 1. Spectrum analyzer SPECTRAN HF-6060 with antenna

The devices D-Link DCS-5222L, Netatmo NSC01-EU, Edimax IC-7113W and ASUS 4GAC55U were chosen for testing (five pieces of each type). Measurements were performed on the chosen systems that examined the intensity and power of their transmission and whether it fully falls within the chosen ISM band.

The wireless transmission for the defined elements was always measured in the way that the device was placed at a 3m distance from the antenna of a SPECTRAN HF-6060 spectrum analyzer. Next, the course of the transmission was recorded by the transmitter cyclically sending a signal (that is why cameras with continuous transmission were chosen) via an ASUS 4G-AC55U router to a cloud disk. The transmitted signal was recorded to the disk and at the same time processed with MSC Spectrum Analyzer software. After each measurement there was always one chart created by the software, which connected all the individual passages within the given measurement. These measurements were repeated in 20 cycles and similar results were always reached, without occurrence of anomalies affecting the measurement. Therefore, only one sample result representing a relevant set is given for each measurement [5].

The process of measuring the intensity of artificial interference was the same as for standard devices. Only the switchboard transmitter was replaced by a low-frequency jammer. After that the maximum intensity value of artificial electromagnetic interference was recorded. This measurement was also evaluated by MSC Spectrum Analyzer software [6; 7].

Further the measurement of natural interference intensity was carried out. The antenna was placed in the middle of a room with windows. Then the mean and minimal intensity values of natural electromagnetic interference were recorded. This measurement was also evaluated by MSC Spectrum Analyzer software [8].

Within the measurement the following values were set on the SPECTRAN HF-6060 spectrum analyzer:

- sample time-20ms;

- samples per cycle - 500;

- bandwidth - $1 \mathrm{MHz}$.

\section{Results and discussion}

There is always only one measurement from one camera type. For all camera types, all waveforms were the same. There is therefore no reason to list all their charts.

A SMART WiFi camera Netatmo NSC01-EU, introduced as a novelty this year, was the first to be tested; besides the capability of recording and wireless transmission in the ISM $2.4 \mathrm{GHz}$ band, it also enables face detection and recognition. Figure 2 shows the intensity of transmission for this camera (represents one example/sample measurement). 


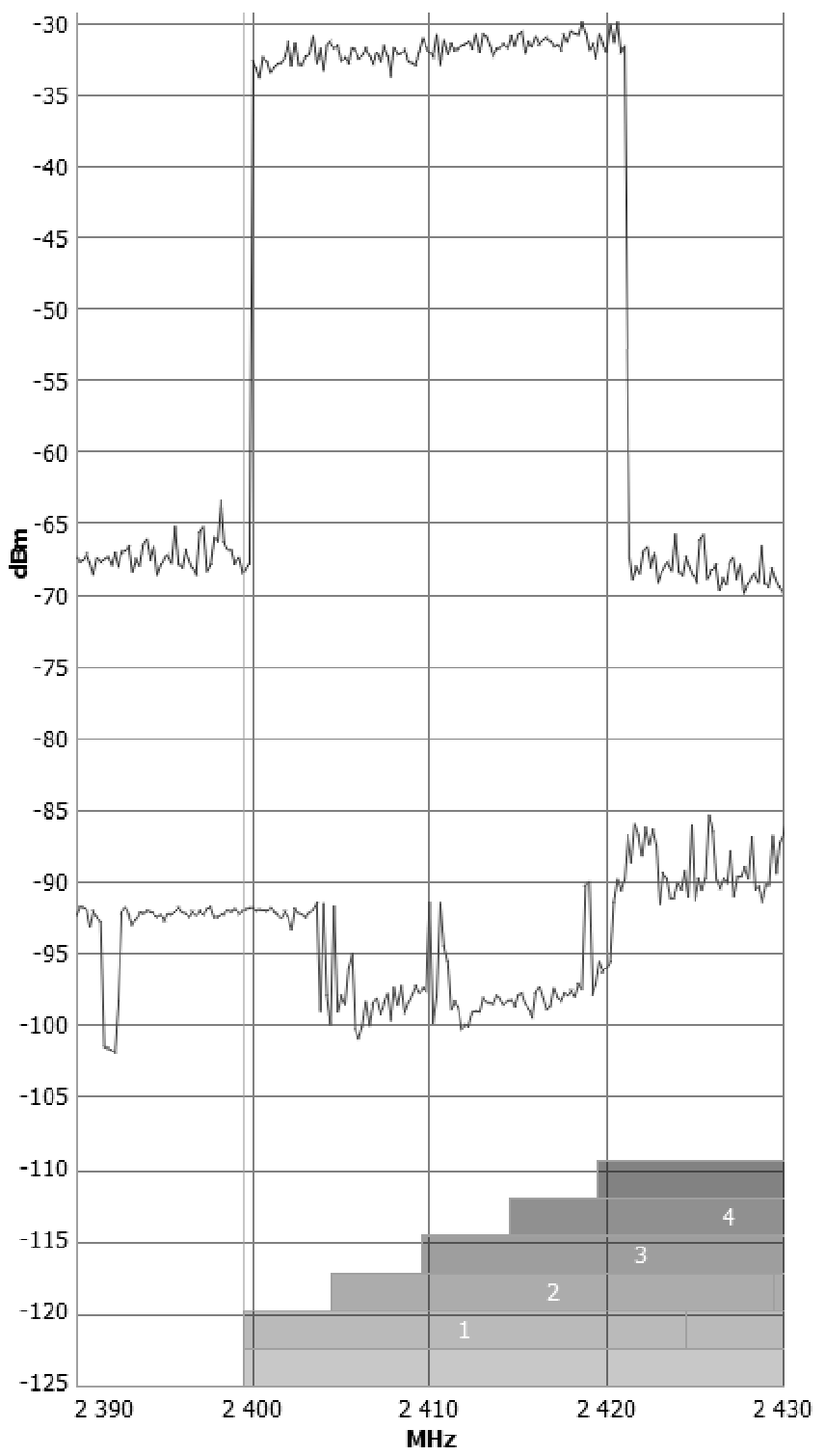

Fig. 2. Wireless transmission at Netatmo NSC01-EU

This camera has a relatively broad and powerful transmission characteristic. The wireless transmission of this camera considerably fills the channel in which it transmits and can be a source of effective interference with wireless transmissions in the relevant WiFi channel. On the other hand, disturbance through natural electromagnetic interference is eliminated and in the case of man-made electromagnetic interference the intensity of the jammer transmission must exceed $-30 \mathrm{dBm}$.

Further a WiFi cloud camera D-Link DCS-5222L was tested, which represents the most frequently occurring type of higher standards. As opposed to the transmissions of a Netatmo NSC01EU camera, the transmission of this camera is significantly weaker (see Fig. 3), however, due to its segmentary way (gradual interrupted transmissions of data packs) of data transmission it loads less the channel in which it broadcasts. In exceptional cases transmission may be affected by natural electromagnetic radiation, but if a tested jammer was used, it would definitely shield this camera transmission.

The last tested WiFi camera Edimax IC-7113W was evidently of lower quality from the first moment of installation. The picture quality through WiFi transmission was not good enough and frequent errors occurred during its transmission with this camera. There were dropouts occurring at a distance greater than $10 \mathrm{~m}$ of the clear line of sight from the router. After measuring its transmission characteristics it turned out that its transmission intensity was very low (see Fig. 4). This camera is 
therefore at a permanent risk of being disturbed by both man-made and natural electromagnetic interference.

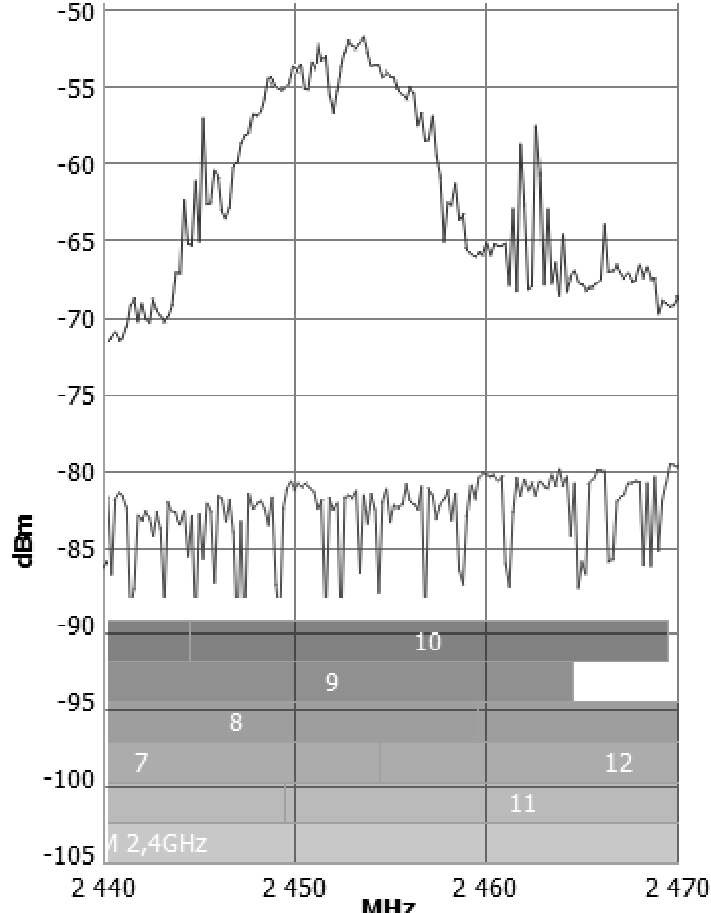

Fig. 3. Wireless transmission at D-Link DCS-5222L

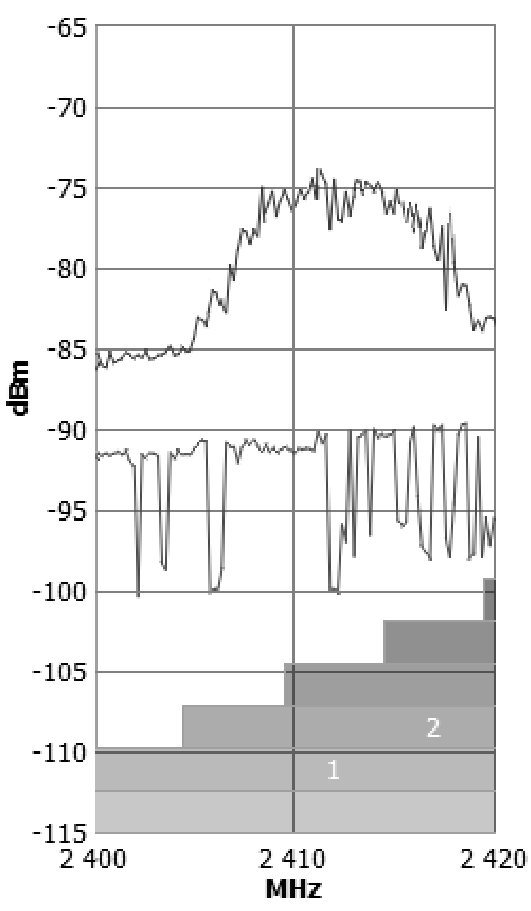

Fig. 4. Wireless transmission at Edimax IC7113W

The measured values of natural electromagnetic interference around the ISM $2.4 \mathrm{GHz}$ band show an average limit of natural electromagnetic interference (Fig. 5). It should not be possible to effect any wireless transmission below this limit. Depending on utilization of individual channels, this limit differs in the different parts of the ISM $2.4 \mathrm{GHz}$ band, as clearly follows from the chart.

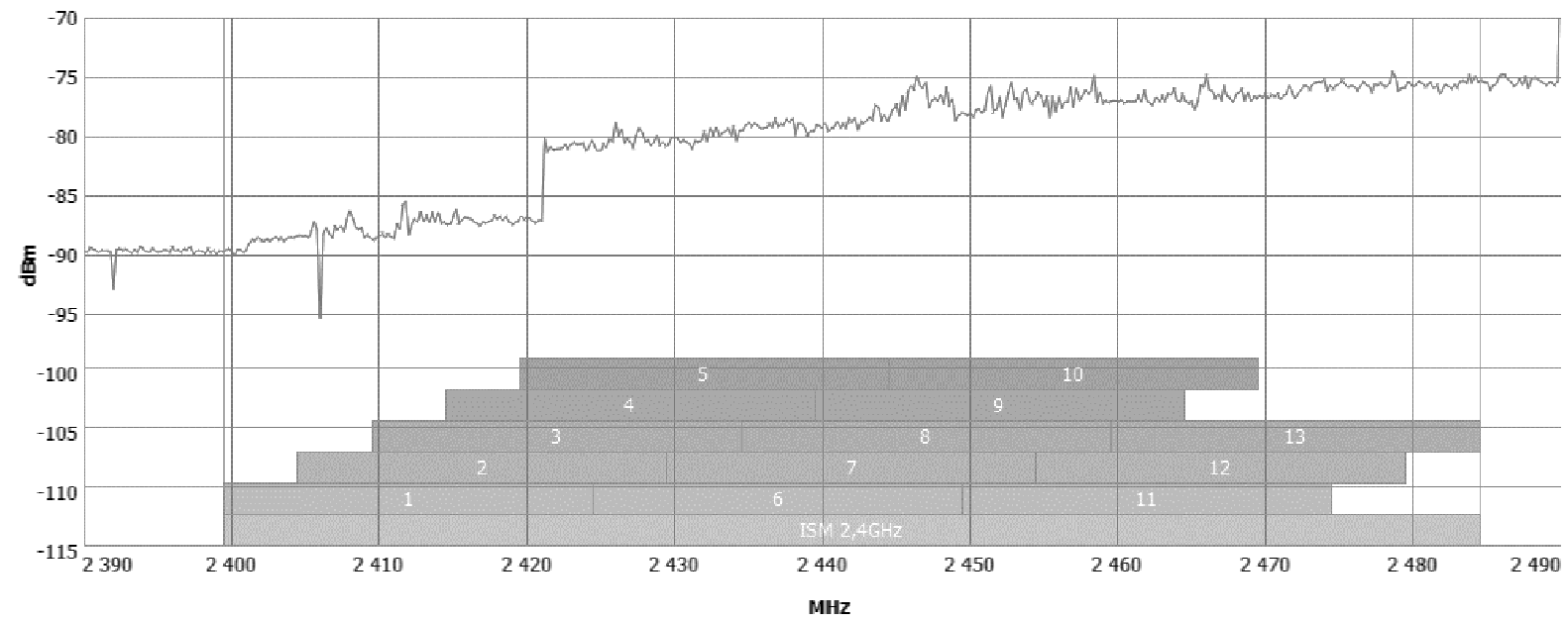

Fig. 4. Intensity of the surrounding natural electromagnetic interference

This research primarily focused on the testing of the ISM $2.4 \mathrm{GHz}$ band, including natural interference. The measured values clearly show that natural electromagnetic interference of wireless transmissions in the measured locality is insignificant. Also at the point, when the maximum interference variation should occur, transmission should not be affected for any of the tested cameras, except for Edimax IC-7113W.

A comparison of different wireless transmissions can be seen in Figure 5, based on the above parameters. This figure shows the percentage of transmission quality in individual measured cameras. 
Weight values for the percentage of multi-criteria analysis of options with an assigned weighting coefficient where this method was modified for the individual types of connection in the way that they were assigned a score for each item on a 100-point scale based on the set parameters. The resulting scores for both methods were then converted to percents and shown in Figure 5. They were selected following the evaluation criteria, which have been selected: wireless bandwidth, wireless power, and the risk of wireless transmission.

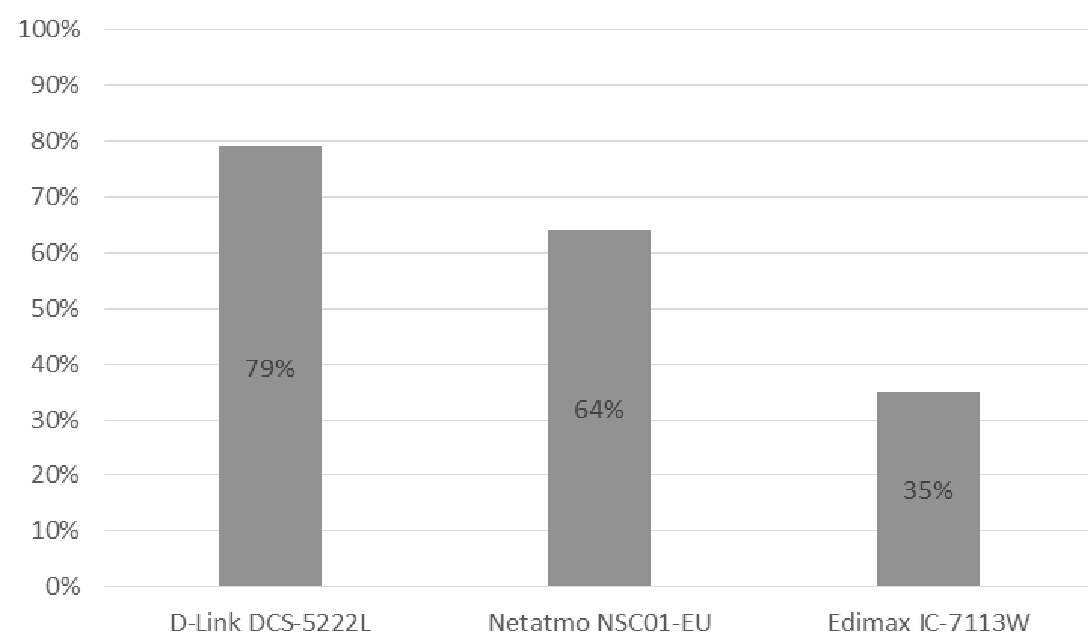

Fig. 5. Evaluation of individual criteria through multi-criteria scoring options

Ever since the first public demonstration of radio and radio connection as carried out by Nikola Tesla in 1893, people have been trying to find some manner of mutual communication which could not be interfered with. We see one of the greatest expansions of this research effort taking place since the Vietnam war, as described by the author of the article "The Progress of Tactical Radios from Legacy Systems to Cognitive Radios" [9]. Many years of research work have passed since this historical turning point. And although natural interference keeps within the limits, it is important to check systems for their ability to withstand it. It is true that natural interference mostly does not exceed the critical limit, however it constantly increases over years and it is just a question of time before it starts widely impact transmissions. The risks related to man-made interference, on the other hand, are much higher, and if an intelligent jammer is used, then not even a system with the highest transmission intensity can withstand. In such case the only protection is the monitoring of wireless communication jamming, which every system should be equipped with by the manufacturer $[9,10]$.

\section{Conclusions}

It follows from the measured characteristics that wireless transmissions of WiFi cameras are resistant to natural interference occurring in the measured locality for all the measured switchboards. The only exception is the Edimax IC-7113W camera, the transmission intensity of which is not sufficient and it is even impacted by natural electromagnetic interference.

Of the measured cameras, D-Link DCS-5222L comes out as the best. Although its transmission characteristic is less powerful, it consequently does not tend to overload a wireless band, as it is the case of the Netatmo NSC01-EU camera.

The use of wireless systems is steadily expanding at present. The use of wireless transmissions defines the modern world today, whether it is mobile phones, WiFi, security systems or other types of wireless systems. What people often do not realize is the fact that the level of electromagnetic interference increases with every next wireless transmission. This malady of the modern technologies has been attracting increasing attention on the part of scientists.

Natural interference may affect wireless transmission and therefore it is important to check systems for their resilience to interference. As mentioned before, it is very important for the systems to have a relevant protection against the band jamming and also to be able to monitor communication loss. When using wireless cameras it is substantial that they have two-way communication, which 
increases the chance of the system to identify the band jamming and at the same time it enables switching to a free band.

\section{Acknowledgements}

It is a project supported by the CULS IGA TF 2016 "The University Internal Grant Agency" (2016:31170/1312/3113).

\section{References}

1. Capel, V.: Security Systems \& Intruder Alarms. Elsevier Science, 1999. 301 p.

2. Křeček, S.: Handbook of security technology. Blatná: Circetus, 2006. 313 s. ISBN 80-902938-24. (in Czech)

3. Petruzzellis T. Alarm Sensor and Security. McGraw-Hill Professional Publishing, 1993. 256 p.

4. Bradna J., Malaták J. Flue gases thermal emission concentration during waste biomass combustion in small combustion device with manual fuel supply, Research in Agricultural Engineering, Vol. 62, Issue 1, 2016, pp. 1-7.

5. Dong Q., Liu D.G, Wright M. Mitigating jamming attacks in wireless broadcast systems, Wireless networks, Vol. 19, Issue: 8, 2013, pp. 1867-1880.

6. Mietzner J., Nickel P., Meusling A., Loos P., Bauch G. Responsive Communications Jamming Against Radio-Controlled Improvised Explosive Devices, IEEE Communications magazine, Vol. 50, Issue: 10, 2012, pp. 38-46.

7. Mpitziopoulos A., Gavalas D., Pantziou G., Konstantopoulos C. Defending wireless sensor networks from jamming attacks, In: $18^{\text {th }}$ international symposium on personal, indoor and mobile radio communications, Vol 1-9, 2007, pp. 81-85.

8. Tahir H., Shah S.A.A. Wireless Sensor Networks - A Security Perspective, In: International multitopic conference, 2008, pp. 189-193.

9. Elmasry G.F. The Progress of Tactical Radios from Legacy Systems to Cognitive Radios, IEEE Communications magazine, Vol. 51, Issue: 10, 2013, pp. 50-56.

10. Yang D.J., Xue G.L., Zhang J., Richa A., Fang X. Coping with a Smart Jammer in Wireless Networks: A Stackelberg Game Approach, IEEE Transactions on wireless communications, Vol. 12, Issue: 8, 2013, pp. 4038-4047. 